\title{
Fabrication of Silicon/Carbon Composite Material with Silicon Waste and Carbon Nanofiber Applied in Lithium-Ion Battery
}

\author{
Ying-Yang Li, Che-Ya Wu, Tzu-Ying Lin*, Jenq-Gong Duh* \\ Department of Materials Science and Engineering, NTHU, Taiwan \\ Email: *tzuying.lin@mx.nthu.edu.tw, ${ }^{*}$ jgd@mx.nthu.edu.tw
}

How to cite this paper: Li, Y.-Y., Wu, C.-Y., Lin, T.-Y. and Duh, J.-G. (2022) Fabrication of Silicon/Carbon Composite Material with Silicon Waste and Carbon Nanofiber Applied in Lithium-Ion Battery. Journal of Environmental Protection, 13, 150-160. https://doi.org/10.4236/jep.2022.131009

Received: December 18, 2021

Accepted: January 16, 2022

Published: January 19, 2022

Copyright $\odot 2022$ by author(s) and Scientific Research Publishing Inc. This work is licensed under the Creative Commons Attribution International License (CC BY 4.0).

http://creativecommons.org/licenses/by/4.0/ (c) (i) Open Access

\begin{abstract}
Silicon ( $\mathrm{Si}$ ) is regarded as a promising material for lithium-ion battery anode because of high theoretical capacity. Nevertheless, Si faces particle pulverization and rapid capacity fading due to serious volume change during the lithiation and the delithiation process. In this work, a silicon/carbon composite constituted to Si powder and carbon nanofiber (CNF) is produced to solve the above issues as a new design structure of anode material. The Si powder was recycled from the silicon slicing waste in photovoltaic industry and the $\mathrm{CNF}$ was from dry rice straws. By mixing the purified Si powder with CNF, the composite was synthesized by the freeze-drying method and calcination. In the cyclic test, Si adding with $1 \mathrm{wt} \% \mathrm{CNF}$ showed $3091 \mathrm{mAh} / \mathrm{g}$ capacity in the first cycle and $1079 \mathrm{mAh} / \mathrm{g}$ capacity after 100 cycles at the current density of $0.5 \mathrm{~A} / \mathrm{g}$, which were both better than pristine Si. SEM images also show the composite structure can eliminate cracks on the surface of the electrode during cycling. CNF attaching on Si particles can increase specific surface area, so binder can easily combine the active materials and the conductive materials together. This strategy enhances the structure stability and prevents the electrode from delamination.
\end{abstract}

\section{Keywords}

Composite Material, Carbon Nanofiber, Waste Silicon, Anode Material, Lithium-Ion Battery

\section{Introduction}

Lithium-ion batteries (LIBs) are widely used in our daily life, including portable electronic devices, electric vehicles, and even grid energy storage [1] [2]. How- 
ever, the manufacturing process and raw materials production also consume energy and raise environmental issues. Thus, recycling and reusing the materials from society is key to protecting our environment [3]. As a result, applying waste silicon recycled from industries such as the photovoltaic (PV) industry in LIBs is of great significance.

To achieve the demand of higher energy density and power density for electric vehicles, silicon $(\mathrm{Si})$ based materials are the most potential candidate for the high theoretical capacity ( $4200 \mathrm{mAh} / \mathrm{g})$, which is about 11 times higher than the conventional graphite capacity $(372 \mathrm{mAh} / \mathrm{g})$ [4] [5]. Nevertheless, Si faces a severe volume change of about $300 \%$ during charging and discharging process, leading to the pulverization of active material and delamination from the current collector. Thus, Si-based materials suffer from the structural degradation and formation of the unstable solid electrolyte interphase (SEI), resulting in rapid capacity decay [6] [7] [8].

To reduce the structural destruction and improve the electrochemical performance of Si-based materials, much research shows that composite designs are effective strategies to offer buffer space for alleviating the volume expansion of Si during lithiation and delithiation process, such as Si/C design [9] [10] and surface modification [11] [12]. Carbon nanofiber (CNF), with great mechanical properties, good conductivity, and large surface area, has been investigated in many lithium-ion batteries studies [13] [14] [15] [16] [17]. The large surface area of CNF can reduce the areal current density and provide more sites for binder to bind electrode material together [17]. Furthermore, both the disordered and graphitic structures of CNF act as active sites for lithium ions transport. CNF can also be a buffer matrix to accommodate the serious volume change of $\mathrm{Si}$ during charging/discharging process, preventing the destruction of the electronic pathway [13] [15]. These characteristics are expected to enhance the electrochemical performances of waste Si for commercial application in lithium-ion batteries.

In this study, Si-CNF composite was synthesized by two steps, including freezedrying method and calcination. The CNF was fabricated from rice crops through a series of chemical treatments. Through the freeze-drying method, Si-CNF composite formed a uniform structure, promoting the attachment of CNF and waste Si particles and improving the electrochemical performances.

\section{Experimental Section}

\subsection{Materials Synthesis}

The raw material of Si was from the slicing waste in PV industry. The HPM solution was used to clean the raw Si powders at $120^{\circ} \mathrm{C}$ for 3 hours. Then Si powder was collected by vacuum suction filter and washed 5 times by DI-water. The sample is denoted as Si-bare. After drying, $6 \mathrm{~g}$ Si and $2 \mathrm{~g}$ PAA were added into $95 \%$ ethanol solution, stirred overnight and heated on the hot plate to evaporate the solvent. The mixture was calcinated at $600^{\circ} \mathrm{C}$ for 3 hours in $\mathrm{Ar} / 5 \% \mathrm{H}_{2}$ with 
the heating rate at $10^{\circ} \mathrm{C} \cdot \mathrm{min}^{-1}$, and the carbon-coated $\mathrm{Si}(\mathrm{Si}-\mathrm{C})$ was produced.

$\mathrm{CNF}$ was fabricated by a series of chemical treatments. First, rice straw was dewaxed with $5 \mathrm{wt} \% \mathrm{NaOH}$ solution. Then $\mathrm{CH}_{3} \mathrm{COOH} / \mathrm{H}_{2} \mathrm{O}_{2}$ solution was used for bleaching and removal of metal impurities. Afterward, TEMPO was adopted to oxidize cellulose and $\mathrm{NaBr}$ was acted as a protectant. Subsequently, CNF solution was synthesized under $500 \mathrm{~W}$ ultra-sonic vibration.

Si-C powder was mixed with $1 \mathrm{wt} \%$ and $2 \mathrm{wt} \% \mathrm{CNF}$ in Di-water for 12 hours. Then the solution was freeze dried for 24 hours and further calcinated at $350^{\circ} \mathrm{C}$ for 3 hours in $\mathrm{Ar} / 5 \% \mathrm{H}_{2}$ with the heating rate at $10^{\circ} \mathrm{C} \cdot \mathrm{min}^{-1}$, and the $\mathrm{Si}-\mathrm{CNF} 1 \mathrm{wt}$ and Si-CNF2wt composites were obtained.

\subsection{Materials Characterization}

The crystal structures of the composite were identified by X-ray diffractometer (XRD, Bruker D2-phaser, $\mathrm{Cu} \mathrm{K} \alpha$ ) at $30 \mathrm{kV}$ and $10 \mathrm{~mA}$. The carbon content was measured by thermal gravimetric analysis (STA 449 F1 Jupiter, Netzsch). The morphology was observed by field-emission SEM (JSM-7600F, JEOL). The surface microstructure was observed by high resolution transmission electron microscopy (HRTEM; JEOL JEM-F200). The surface area was measured by BET surface area analyzers (ASAP 2020 V4.02).

\subsection{Electrochemical Characterization}

CR2032-type coin cells were used to test electrochemical performance. The anode materials were prepared with $60 \mathrm{wt} \%$ active material, $20 \mathrm{wt} \%$ carbon black, and 20 wt\% Polyacrylic acid (PAA) binder. Slurry containing these three materials in 95 vol\% ethanol was blended homogeneously and coated on $20 \mu \mathrm{m} \mathrm{Cu}$ foils, then the electrodes were completely dried under vacuum for $3 \mathrm{~h}$. The coin cells were assembled in an Ar-filled glovebox, where the contents of $\mathrm{H}_{2} \mathrm{O}$ and $\mathrm{O}_{2}$ were less than $0.1 \mathrm{ppm}$, containing electrodes, Li-metal, polypropylene separator, and electrolyte composed of 1.0 $\mathrm{M} \mathrm{LiPF}_{6}$ in EC/DEC (1:1, vol\%).

The electrochemical tests of half cells were performed in the voltage range of $0.01 \mathrm{~V}$ and $1.5 \mathrm{~V}$ vs. $\mathrm{Li} / \mathrm{Li}^{+}$. Galvanostatic charge/discharge measurements were operated using Arbin battery tester. Cyclic voltammetry (CV) was carried out at a scan rate of $0.1 \mathrm{mV} / \mathrm{s}$.

\section{Results and Discussion}

\subsection{Morphology and Material Characterization}

To detect the crystal structure of the samples, XRD analysis was measured. Figure 1 reveals the XRD patterns of Si-bare, Si-CNF1wt, and Si-CNF2wt. Si-bare displays high crystallinity without other phases, indicating that the impurity was successfully removed via HPM solution. After calcination at $350^{\circ} \mathrm{C}$, the XRD patterns of Si-CNF1wt and Si-CNF2wt still remain the high crystallinity of Si-bare without extra phase formation. Figure 2 shows the Thermogravimetric analysis (TGA) results of the Si-CNF1wt and Si-CNF2wt for the determination 


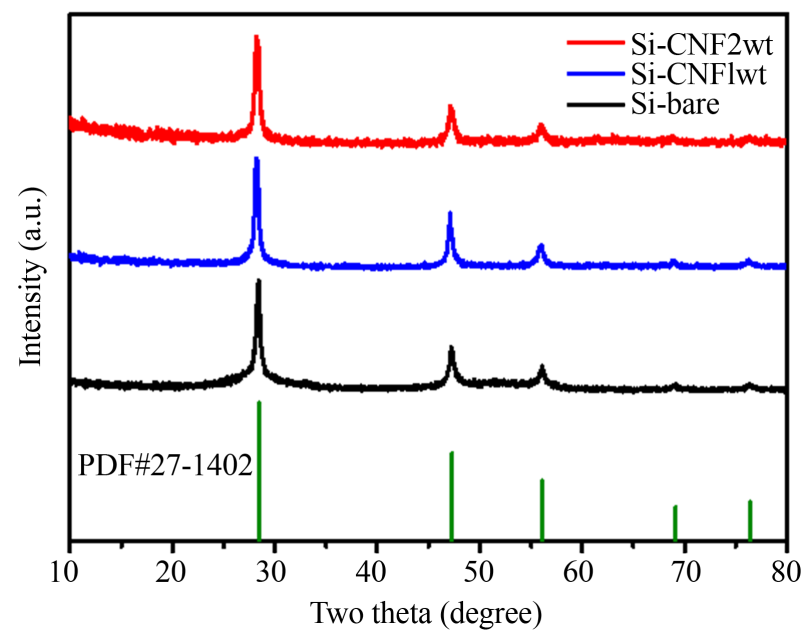

Figure 1. XRD diffraction of Si-bare, Si-CNF1wt, Si-CNF2wt.

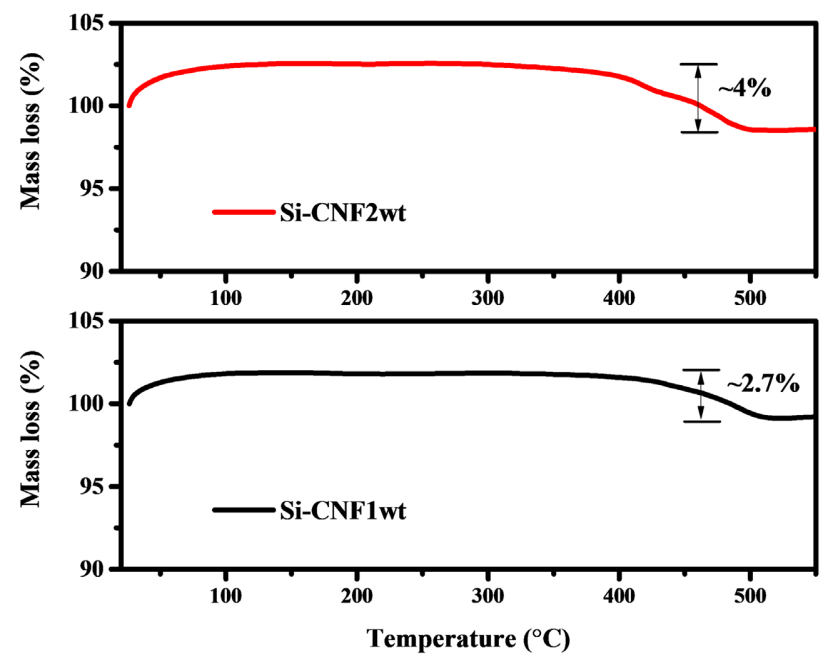

Figure 2. TGA of Si-CNF1wt and Si-CNF2wt sintered from $25^{\circ} \mathrm{C}$ to $550^{\circ} \mathrm{C}$ in air atmosphere.

of carbon content. The temperature was conducted from room temperature up to $600^{\circ} \mathrm{C}$ in air. The weight loss of Si-CNF1wt and Si-CNF2wt is $2.7 \%$ and $4 \%$, respectively.

To further investigate the structure of CNF, Raman spectroscopy was performed (Figure 3). The peaks around 1382 and near $1568 \mathrm{~cm}^{-1}$ were designated as D-band and G-band, respectively. D-band is associated to the defects and disordered carbon, whereas G-band is corresponding to graphitized carbon [18]. The integrated intensity ratio, $\mathrm{I}_{\mathrm{D}} / \mathrm{I}_{\mathrm{G}}$, represents the degree of structural defects, arising from edges and disorder carbon atoms. Thus, the higher ratio is favorable for lithium ion insertion [19] [20]. The value of $I_{D} / I_{G}$ for $C N F$ is 0.65 , demonstrating that defects were induced during the pyrolysis of cellulose. Defects in the CNF offer pathways for lithium ions to transport during charging-discharging process, enhancing the diffusivity of lithium ions and conductivity of active materials [21]. 


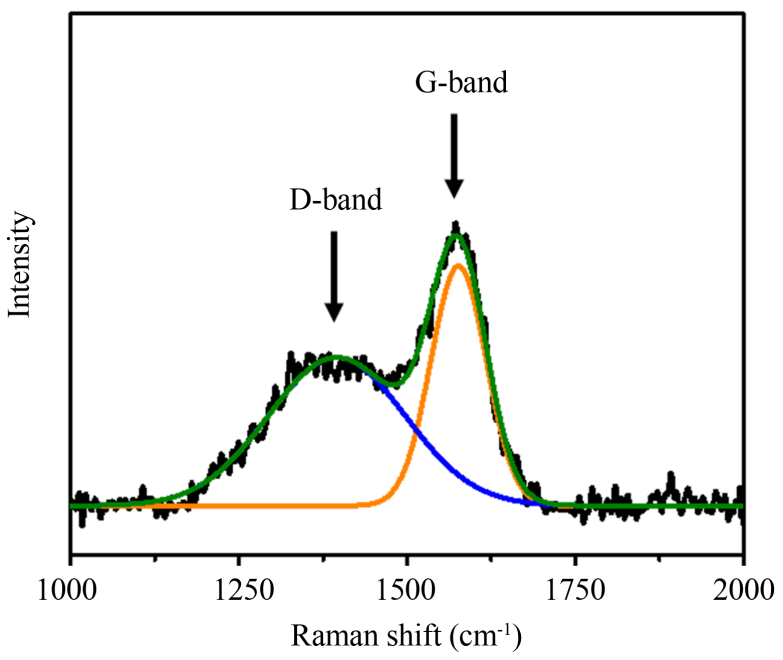

Figure 3. Raman patterns of cellulose nanofiber after calcination.

The morphology of materials is shown via SEM and TEM. Figures 4(a)-(c) are SEM images of Si-bare, Si-CNF1wt, and Si-CNF2wt, respectively. From the image of Si-bare, the shape of waste $\mathrm{Si}$ was irregular and the size distribution was around $100 \mathrm{~nm}$ to $1 \mu \mathrm{m}$. In Figure 4(b) and Figure 4(c), CNF was attached on the surface of Si particles. To investigate the surface morphologies of Si-CNF1wt, high-resolution TEM (HR-TEM) was performed, and images are shown in Figure 4(d) and Figure 4(e). TEM images display a uniform carbon-coating layer with the thickness around $5 \mathrm{~nm}$ on the surface of Si particles. Figure 4(e) shows that CNF was attached to the Si particles, similar to the image shown in Figure 4(b). Further investigation of the surface area was conducted by BET measurement. In Table 1, the surface area of composites and pristine shows $12.16 \mathrm{~m}^{2} / \mathrm{g}$, $13.02 \mathrm{~m}^{2} / \mathrm{g}$, and $1.64 \mathrm{~m}^{2} / \mathrm{g}$ for Si-CNF1wt, Si-CNF2wt and Si-bare, respectively, indicating that the micropore surface area of composites are higher than Si-bare. The SEM and TEM results also present that CNF was uniform attached to Si. The increased surface area by CNF may create more sites for binder to connect with active materials and conductive materials, strengthening the structural stability.

\subsection{Electrochemical Measurements}

Cycling tests at the current density of $0.5 \mathrm{~A} / \mathrm{g}$ for Si-bare, Si-CNF1wt, and SiCNF2wt composites are shown in Figure 5(a). The discharge capacities and coulombic efficiency of first cycle for Si-bare were $2317 \mathrm{mAh} / \mathrm{g}$ and $87.5 \%$, respectively. After adding CNF in the materials, the first cycle of discharge capacities for Si-CNF1wt and Si-CNF2wt were $3091 \mathrm{mAh} / \mathrm{g}$ and $2861 \mathrm{mAh} / \mathrm{g}$. The first cycle of coulombic efficiency for Si-CNF1wt and Si-CNF2wt were also increased to $88.4 \%$ and $90.4 \%$. After 50 cycles, Si-CNF1wt exhibited a better cycling stability and the capacity remained $1691 \mathrm{mAh} / \mathrm{g}$, while Si-CNF2wt and Si-bare showed $916 \mathrm{mAh} / \mathrm{g}$ and $281 \mathrm{mAh} / \mathrm{g}$. The improved cycling performances are attributed to the addition of CNF, creating high surface area for binding network 


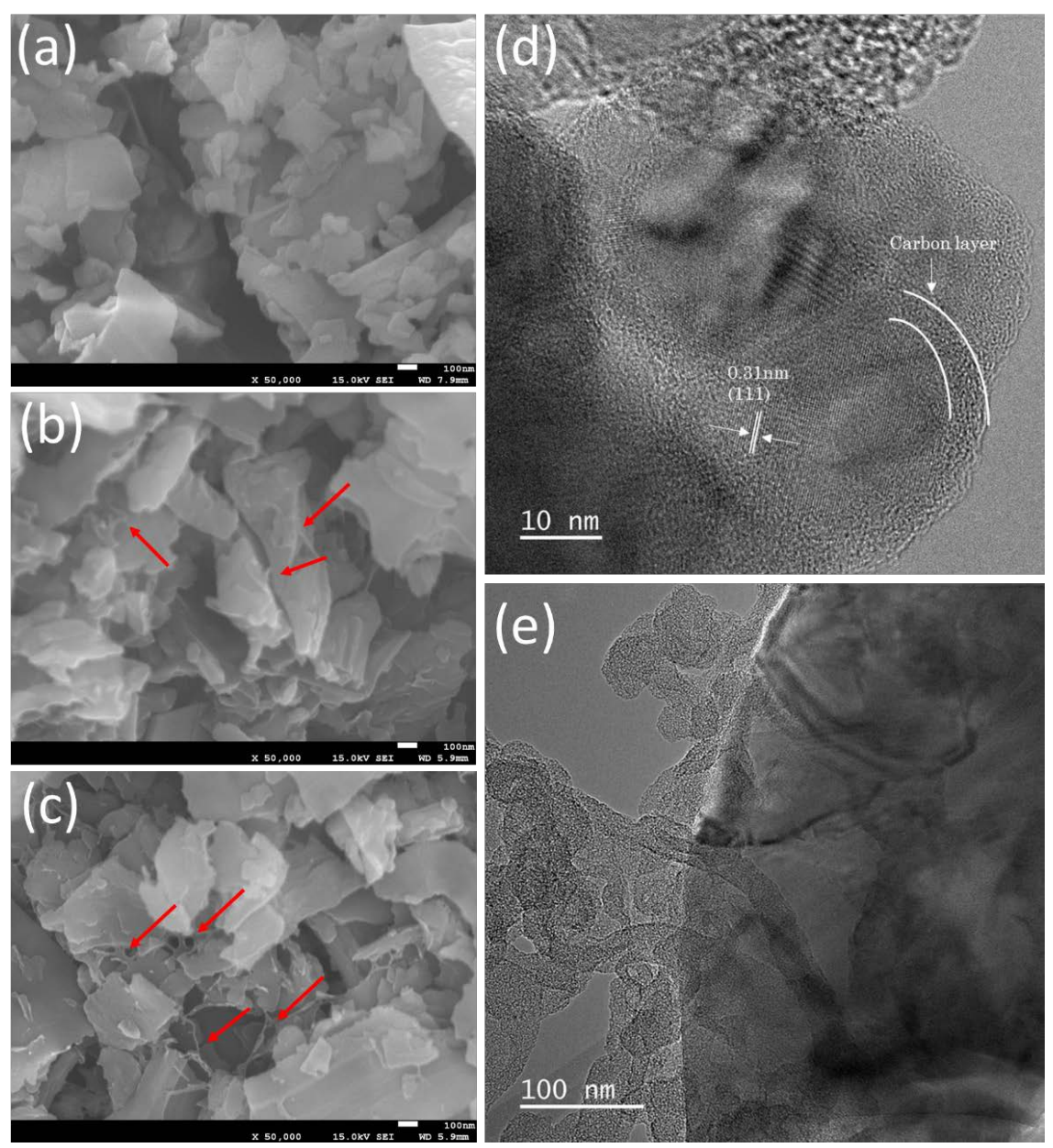

Figure 4. SEM image of (a) Si-bare, (b) Si-CNF1wt, (c) Si-CNF2wt, and TEM image of (d) (e) Si-CNF1wt.

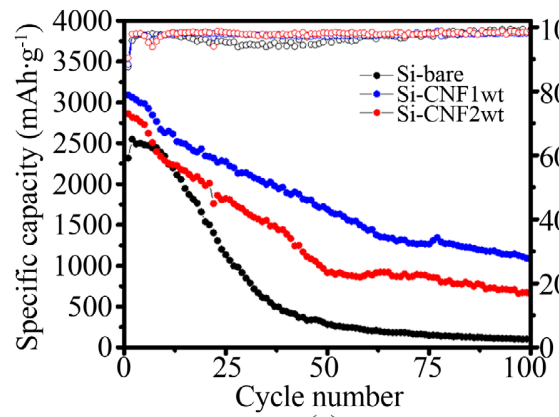

(a)

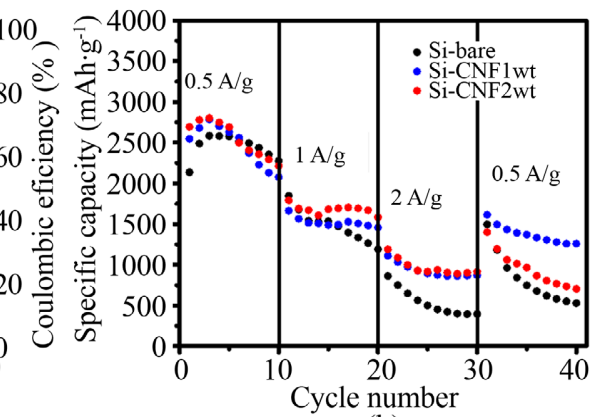

(b)

Figure 5. (a) Cycle performance at $0.5 \mathrm{~A} / \mathrm{g}$; (b) C-rate performance.

Table 1. BET surface area of Si-bare, Si-CNF1wt, and Si-CNF2wt.

\begin{tabular}{ccc}
\hline Material & Total surface area $\left(\mathrm{m}^{2} / \mathrm{g}\right)$ & Micropore surface area $\left(\mathrm{m}^{2} / \mathrm{g}\right)$ \\
\hline Si-bare & 1.64 & 2.34 \\
Si-CNF1wt & 12.16 & 8.78 \\
Si-CNF2wt & 13.02 & 6.41 \\
\hline
\end{tabular}


among active materials, conductive materials and binder. CNF provides a buffer space and acts as a connecting agent to combine electrode materials together. As a result, severe volume change of Si during lithiation and delithiation and the structural destruction of electrode material are mitigated. However, the CNF addition should be controlled in appropriate ratio. Si-CNF2wt composite demonstrates worse retention than Si-CNF1wt. The reason is that the content of PAA binder in the electrode was not enough to capture all the CNF for the overincreased surface area. Consequently, the ratio of CNF ought to be well designed in the material.

Rate performance at different current densities is also used to evaluate anode stability and performance as shown in Figure 5(b). The current density was changed from $0.5,1,2$, to $0.5 \mathrm{~A} / \mathrm{g}$ in every 10 cycles in order. The specific capacities of Si-bare, Si-CNF1wt, and Si-CNF2wt were respectively 2136, 2547, and 2694 at the first 10 cycles under $0.5 \mathrm{~A} / \mathrm{g}, 1846,1664$, and 1790 at cycle 10 to 20 under $1 \mathrm{~A} / \mathrm{g}, 861,1109$, and 1188 at cycle 20 to 30 under $2 \mathrm{~A} / \mathrm{g}$, and 1495, 1613, and $1400 \mathrm{mAh} / \mathrm{g}$ at cycle 30 to 40 under $0.5 \mathrm{~A} / \mathrm{g}$. Si-CNF1wt and Si-CNF2wt composites demonstrate a superior rate performance than Si-bare at $2 \mathrm{~A} / \mathrm{g}$ since CNF defects may decrease the transferring energy barrier. Furthermore, Si-CNF1wt exhibited a greater rate performance and lower specific capacity loss than Si-bare and $\mathrm{Si}-\mathrm{CNF} 2 \mathrm{wt}$ at the second-time $0.5 \mathrm{~A} / \mathrm{g}$, indicating that Si-CNF1wt acquires better stability performance after a long cycle and high C-rate test.

Figure 6 is the CV plot at the scan rate of $0.1 \mathrm{mV} / \mathrm{s}$ for Si-bare, Si-CNF1wt, and Si-CNF2wt among the voltage window of $0.01-1.5 \mathrm{~V}$. During the lithiation process, the reduction current increased below $0.2 \mathrm{~V}$ in the first sweep cycle in all electrodes. This may be contributed to the transformation of crystalline-Si to poly-crystalline $\mathrm{Li}_{22} \mathrm{Si}_{5}$. In the delithiation process of the first cycle, the peaks at $0.36 \mathrm{~V}$ and $0.54 \mathrm{~V}$ were related to a partial Li-discharge to $\mathrm{Li}_{13} \mathrm{Si}_{4}$ and full Li-discharge to amorphous Si (Figure 6(a)) [22] [23]. It is found that the addition of CNF decreased the polarization in the first cycle, attributing to the defects in CNF. For the second cycle (Figure 6(b)), an additional cathodic peak at about $0.21 \mathrm{~V}$ for Si-bare and $0.17 \mathrm{~V}$ for Si-CNF1wt and Si-CNF2wt was corresponding to Li-Si alloy formation [24].

To further exhibit the benefits of the CNF in waste $\mathrm{Si}$, the SEM images before cycling and after 50 cycles for Si-bare and Si-CNF1wt electrodes are displayed in Figure 7. Before cycling, all electrodes showed a smooth surface and similar structure (Figures 7(a)-(c)); nevertheless, the Si-bare electrode was severely pulverized with large cracks up to $10 \mu \mathrm{m}$ after 50 cycles (Figure 7 (d)). In contrast, Si-CNF1wt and Si-CNF2wt retained good integrity with few micro cracks on the surface. Also, the crack formation on Si-CNF1wt electrode (Figure 7(e)) was less than Si-CNF2wt (Figure 7(f)) after 50 cycles. The morphology is consistent with the performances in the cyclic tests and rate performances. Si-CNF1wt reveals a stable structure and $\mathrm{CNF}$ prevents the $\mathrm{Si}$ particles from pulverization during electrochemical tests. 


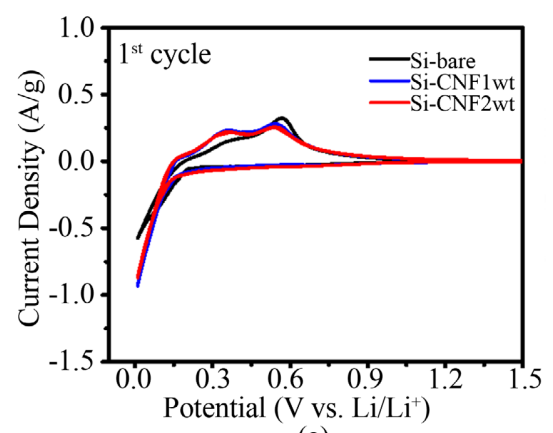

(a)

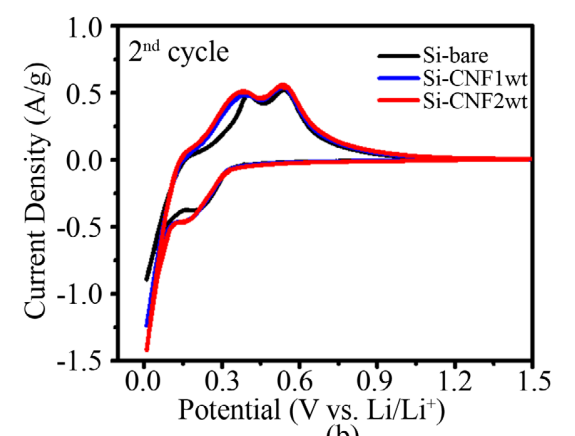

(b)

Figure 6. CV plot of Si-bare, Si-CNF1wt, Si-CNF2wt at the scan rate of $0.1 \mathrm{mV} / \mathrm{s}$. (a) First cycle; (b) Second cycle.
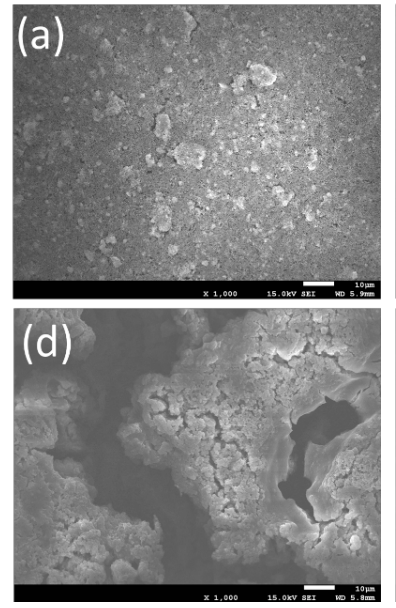
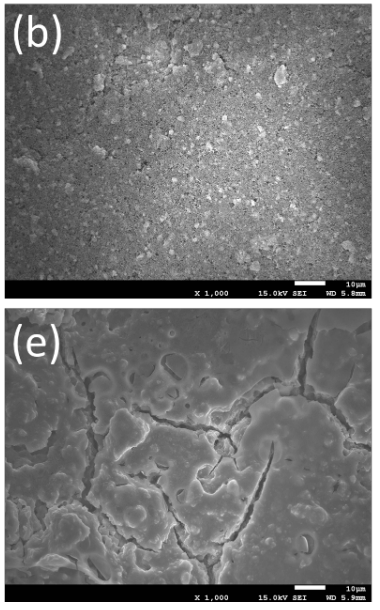
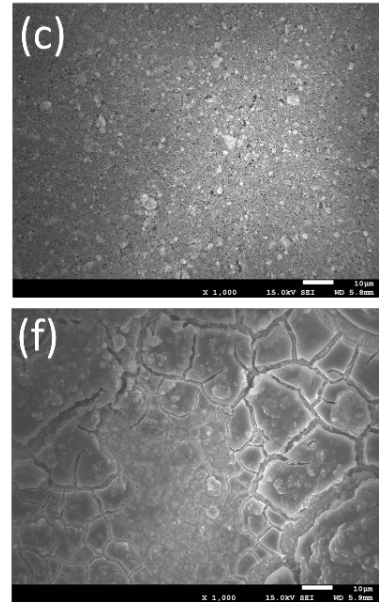

Figure 7. SEM images of electrodes morphologies before cycling (a) Si-bare; (b) Si-CNF1wt; (c) Si-CNF2wt; after 50 cycles; (d) Si-bare; (e) Si-CNF1wt; (f) Si-CNF2wt.

\section{Conclusion}

This work not only successfully recycled waste silicon from the photovoltaic industry, but also enhanced the electrochemical performance by CNF addition and structural design. Composite of waste silicon and CNF anode was successfully fabricated by freeze-drying method and further calcination. This method provided a uniform dispersion of CNF and Si particles. The composite shows excellent electrochemical performances with stable retention in the cycle test and great rate capability than pristine waste $\mathrm{Si}$. After 50 cycles, Si-CNF1wt remained $1691 \mathrm{mAh} / \mathrm{g}$, while Si-bare only displayed $281 \mathrm{mAh} / \mathrm{g}$. Moreover, Si-CNF1wt also demonstrated a higher capacity $(1109 \mathrm{mAh} / \mathrm{g})$ than Si-bare $(861 \mathrm{mAh} / \mathrm{g})$ in $\mathrm{C}$-rate test at the current rate of $2 \mathrm{~A} / \mathrm{g}$. This indicates that the enhanced performances are attributed to the moderate addition of CNF in waste silicon which increases the surface area of the particles, forming a firm binding network to strengthen the electrode integrity. However, excessive addition of CNF in waste silicon has a limited improvement of the performances, owing to the overincreased surface area, making binder fail to stretch to all the materials. Overall, this study provides green manufacturing of recycling and synthesis for the com- 
posite of CNF and waste Si applied in the next-generation lithium-ion battery.

\section{Acknowledgements}

Financial supports from the Ministry of Science and Technology, Taiwan, under Contract No. MOST 109-2622-E-007-020 and MOST 110-2636-E-007-015 are much appreciated. The authors would also like to thank the technical support from the instrument manager of HRTEM (Ms. Yi-Jen Yu).

\section{Authors' Contributions}

Ying-Yang Li: Conceptualization, methodology, formal analysis, investigation, writing original draft.

Che-Ya Wu: Conceptualization, formal analysis, validation.

Tzu-Ying Lin: Supervision, writing, review \& editing.

Jenq-Gong Duh: Project administration, supervision, writing review \& editing.

\section{Conflicts of Interest}

There are no conflicts to declare.

\section{References}

[1] Su, M., Wan, H., Liu, Y., Xiao, W., Dou, A., Wang, Z. and Guo, H. (2018) MultiLayered Carbon Coated Si-Based Composite as Anode for Lithium-Ion Batteries. Powder Technology, 323, 294-300. https://doi.org/10.1016/j.powtec.2017.09.005

[2] Sun, J., Shi, J., Ban, B., Li, J., Wei, M., Wang, Q. and Chen, J. (2019) Porous Si/C Anode Materials by Al-Si Dealloying Method with PEA Surfactant Assisted CrossLinked Carbon Coating for Lithium-Ion Battery Applications. Electrochimica Acta, 327, Article ID: 134995. https://doi.org/10.1016/j.electacta.2019.134995

[3] Ruan, D., Wu, L., Wang, F., Du, K., Zhang, Z., Zou, K., Wu, X. and Hu, G. (2021) A Low-Cost Silicon-Graphite Anode Made from Recycled Graphite of Spent LithiumIon Batteries. Journal of Electroanalytical Chemistry, 884, Article ID: 115073. https://doi.org/10.1016/j.jelechem.2021.115073

[4] Xu, Y., Yin, G., Ma, Y., Zuo, P. and Cheng, X. (2010) Nanosized Core/Shell Silicon@Carbon Anode Material for Lithium Ion Batteries with Polyvinylidene Fluoride as Carbon Source. Journal of Materials Chemistry, 20, 3216-3220. https://doi.org/10.1039/b921979j

[5] Di, F., Zhou, W., Yang, H., Sun, C., Geng, X., Chen, Y., Li, L., Liu, Z. and An, B. (2021) Surface Modification and Functional Structure Space Design to Improve the Cycle Stability of Silicon Based Materials as Anode of Lithium Ion Batteries. Coatings, 11, Article No. 1047. https://doi.org/10.3390/coatings11091047

[6] Liu, W., Xu, H., Qin, H., Lv, Y., Zhu, G., Lei, X., Lin, F., Zhang, Z. and Wang, L. (2020) Rapid Coating of Asphalt to Prepare Carbon-Encapsulated Composites of Nano-Silicon and Graphite for Lithium Battery Anodes. Journal of Materials Science, 55, 4382-4394. https://doi.org/10.1007/s10853-019-04313-x

[7] Liu, Q., Hu, X., Liu, Y. and Wen, Z. (2020) One-Step Low-Temperature Molten Salt Synthesis of Two-Dimensional Si@SiOx@C Hybrids for High-Performance LithiumIon Batteries. ACS Applied Materials \& Interfaces, 12, 55844-55855. https://doi.org/10.1021/acsami.0c15882 
[8] Zhou, X., Wu, L., Yang, J., Tang, J., Xi, L. and Wang, B. (2016) Synthesis of NanoSized Silicon from Natural Halloysite Clay and Its High Performance as Anode for Lithium-Ion Batteries. Journal of Power Sources, 324, 33-40. https://doi.org/10.1016/j.jpowsour.2016.05.058

[9] Kim, J.-C., Kim, K.-J. and Lee, S.-M. (2021) Preparation and Characterization of Core-Shell Structure Hard Carbon/Si-Carbon Composites with Multiple Shell Structures as Anode Materials for Lithium-Ion Batteries. Energies, 14, Article No. 2104. https://doi.org/10.3390/en14082104

[10] Xu, Q., Li, J.-Y., Sun, J.-K., Yin, Y.-X., Wan, L.-J. and Guo, Y.-G. (2017) WatermelonInspired Si/C Microspheres with Hierarchical Buffer Structures for Densely Compacted Lithium-Ion Battery Anodes. Advanced Energy Materials, 7, Article ID: 1601481. https://doi.org/10.1002/aenm.201601481

[11] Zhu, Y., Hu, W., Zhou, J., Cai, W., Lu, Y., Liang, J., Li, X., Zhu, S., Fu, Q. and Qian, Y. (2019) Prelithiated Surface Oxide Layer Enabled High-Performance Si Anode for Lithium Storage. ACS Applied Materials \& Interfaces, 11, 18305-18312. https://doi.org/10.1021/acsami.8b22507

[12] Alaboina, P.K., Cho, J.-S., Uddin, M.-J. and Cho, S.-J. (2017) Mechanically Prelithiated Silicon Nano Alloy as Highly Engineered Anode Material. Electrochimica Acta, 258, 623-630. https://doi.org/10.1016/j.electacta.2017.11.107

[13] Howe, J.Y., Burton, D.J., Qi, Y., Meyer, H.M., Nazri, M., Nazri, G.A., Palmer, A.C. and Lake, P.D. (2013) Improving Microstructure of Silicon/Carbon Nanofiber Composites as a Li Battery Anode. Journal of Power Sources, 221, 455-461. https://doi.org/10.1016/j.jpowsour.2012.08.026

[14] Li, W., Li, M., Shi, J.A., Zhong, X., Gu, L. and Yu, Y. (2018) Carbon Nanofiber interlayer: A Highly Effective Strategy to Stabilize Silicon Anodes for Use in LithiumIon Batteries. Nanoscale, 10, 12430-12435. https://doi.org/10.1039/C8NR03623C

[15] Si, Q., Hanai, K., Ichikawa, T., Hirano, A., Imanishi, N., Takeda, Y. and Yamamoto, O. (2010) A High Performance Silicon/Carbon Composite Anode with Carbon Nanofiber for Lithium-Ion Batteries. Journal of Power Sources, 195, 1720-1725. https://doi.org/10.1016/j.jpowsour.2009.09.073

[16] Chen, Y., Li, X., Park, K., Song, J., Hong, J., Zhou, L., Mai, Y.W., Huang, H. and Goodenough, J.B. (2013) Hollow Carbon-Nanotube/Carbon-Nanofiber Hybrid Anodes for Li-Ion Batteries. Journal of the American Chemical Society, 135, 16280-16283. https://doi.org/10.1021/ja408421n

[17] Zhang, A., Fang, X., Shen, C., Liu, Y. and Zhou, C. (2016) A Carbon Nanofiber Network for Stable Lithium Metal Anodes with High Coulombic Efficiency and Long Cycle Life. Nano Research, 9, 3428-3436. https://doi.org/10.1007/s12274-016-1219-2

[18] Fei, L., Williams, B.P., Yoo, S.H., Kim, J., Shoorideh, G. and Joo, Y.L. (2016) Graphene Folding in Si Rich Carbon Nanofibers for Highly Stable, High Capacity Li-Ion Battery Anodes. ACS Applied Materials \& Interfaces, 8, 5243-5250. https://doi.org/10.1021/acsami.5b10548

[19] Yoon, Y.S., Jee, S.H., Lee, S.H. and Nam, S.C. (2011) Nano Si-Coated Graphite Composite Anode Synthesized by Semi-Mass Production Ball Milling for Lithium Secondary Batteries. Surface and Coatings Technology, 206, 553-558. https://doi.org/10.1016/j.surfcoat.2011.07.076

[20] Zhang, S., Du, H., He, J., Huang, C., Liu, H., Cui, G. and Li, Y. (2016) Nitrogen-Doped Graphdiyne Applied for Lithium-Ion Storage. ACS Applied Materials \& Interfaces, 8, 8467-8473. https://doi.org/10.1021/acsami.6b00255 
[21] Wang, W., Favors, Z., Li, C., Liu, C., Ye, R., Fu, C., Bozhilov, K., Guo, J., Ozkan, M. and Ozkan, C.S. (2017) Silicon and Carbon Nanocomposite Spheres with Enhanced Electrochemical Performance for Full Cell Lithium Ion Batteries. Scientific Reports, 7, Article No. 44838. https://doi.org/10.1038/srep44838

[22] Kang, K., Lee, H.-S., Han, D.-W., Kim, G.-S., Lee, D., Lee, G., Kang, Y.-M. and Jo, M.-H. (2010) Maximum Li Storage in Si Nanowires for the High Capacity ThreeDimensional Li-Ion Battery. Applied Physics Letters, 96, Article No. 053110. https://doi.org/10.1063/1.3299006

[23] Pereira-Nabais, C., Światowska, J., Chagnes, A., Ozanam, F., Gohier, A., Tran-Van, P., Cojocaru, C.-S., Cassir, M. and Marcus, P. (2013) Interphase Chemistry of Si Electrodes Used as Anodes in Li-Ion Batteries. Applied Surface Science, 266, 5-16. https://doi.org/10.1016/j.apsusc.2012.10.165

[24] Jiang, T., Zhang, S., Qiu, X., Zhu, W. and Chen, L. (2007) Preparation and Characterization of Silicon-Based Three-Dimensional Cellular Anode for Lithium Ion Battery. Electrochemistry Communications, 9, 930-934.

https://doi.org/10.1016/j.elecom.2006.11.031 